tragus $0^{\prime \prime} \cdot 3 \times 0^{\prime \prime} \cdot 15$; forearm $1^{\prime \prime} \cdot 95$; thumb $0^{\prime \prime} \cdot 55$; third fingermetacarp. $1^{\prime \prime} \cdot 4$, 1st ph. $l^{\prime \prime} \cdot 0$, 2nd ph. $1^{\prime \prime} \cdot 2$; fifth finger-metacarp. $1^{\prime \prime} \cdot 65$, 1st ph. $0^{\prime \prime} \cdot 55$, 2nd ph. $0^{\prime \prime} \cdot 55$; tibia $0^{\prime \prime} \cdot 95$; calcaneum $0^{\prime \prime} \cdot 7$; foot $0^{\prime \prime} \cdot 45$.

\title{
2. Notice sur quelques Coquilles du Pérou.
}

Par le Prince Ladislas Lubomirski.

[Received October 7, 1879.]

\section{(Plates LV. \& LVI.)}

Les deux naturalistes polonais, MM. Jelski et Stolzmann, qui ont fait l'exploration du Haut-Pérou, l'un depuis 1870 jusqu'à 1874 , l'autre depuis 1875 jusqu'à 1878 , ont envoyé de riches collections au Musée de Varsovie; entre autres objets zoologiques, ils nous ont procuré de ces contrées différentes espèces de coquilles terrestres, les unes déjà connues, d'autres encore inédites, que je me propose de publier dans ces notices. La description détaillée des localités qu'ils ont parcourues, a été déjà mainte fois consignée dans les Listes des Oiseaux du Pérou, par le Conservateur du Musée de Varsovie, M. Taczanowski, et publiée dans les 'Proceedings' de la Société.

1. Succinea peruviana, Phil.

Succinea peruviana, Phil., Monogr. Helic. viv. Pfr. t. v. p. 38.

Lima, envoyée par M. Jelski en 1871.

2. Helix (Ammonoceras) trochilioneides, D'Orb.

Helix (Ammonoceras) trochilioneides, D’Orb., Monogr. Helic. viv. Pfr. t. i. p. 113.

Lima, envoyée par M. Jelski en 1871.

3. Helix (Polita) santanaËnsis, Pfr.

Helix (Polita) santanaënsis, Pfr., Monogr. Helic. viv. Pfr. t. iv. p. 82.

Tambillo, envoyée par M. Stolzmann en 1878.

4. Helix (Systrophia) pseudo-planorbis, n. sp. (Plate LV. figs. 1, 2, 3.)

Species Helici gyrellæ, Mor., affinis. Testa latissime umbilicata, planorboidea, oblique confertim striata, albida, epidermide lutescente obtecta; spira fere plana, sutura profunda ; anfr. 6-7, ultimus vix depressus, non descendens; apert. diagonalis suboblique rotundata ; perist. simplex, margine columellari regulariter arcuato.

Diam. maj. $16 \frac{1}{2}$, min. 8 , alt. 3 mill.

Pujupé, entre Hualguayoc et Chota, à 10,000 pieds d'altitude, trouvée sous un tronc d'arbre mort, envoyée par M. Stolzmann au nombre d'une cinquantaine d'exemplaires en 1878 . 
Diffère de l' Helix gyrella, Mor., par sa dimension, par sa contexture plus ferme, par sa couleur, qui est blanchâtre, et par son épiderme jaunâtre qui le recouvre, par sa spire, qui est tant soit peu bombée, et par le nombre des tours.

Diffère de l'Helix tortilis, Mor., par sa dimension, par ses stries, qui dans le nôtre sont réparties régulièrement sur tous les tours, par sa hauteur et par le manque du sinus au bord supérieur de l'ouverture.

5. Helix (Lysinoë) alsophila, Phil.

Helix (Lysinoë) alsophila, Phil., Monogr. Helic. viv. Pfr. t. v. p. 303.

Chota, envoyée par M. Stolzmann en 1878. $5,6$.

6. Helix (Isomeria) stolzmanni, n. sp. (Plate LV. figs. 4,

Testa semiobtecta, umbilicata, depressa, solida, striata, striis obliquis minutissime granulatis; saturate castanea; spira convexiuscula, vix elevata; anfractus regulariter accrescentes, ultimus carinatus, antice defleasus, basi versus aperturam inflatus; apert. perobliqua, parva, securiformis ; perist. purpureo-fulvescens, incrassatum, reflexum, ad carinam angulatum, marginibus callo albo funiculato junctis, dente unico coniformi infra carinam posito.

Diam. maj. 40 , min. 33, alt. 17 mill,

Montaña de Palto, près de Tambillo, district de Chota, trouvées par M. Stolzmann au mois de Mai 1877, un exemplaire vivant, ayant toutes ses couleurs, et 4 exemplaires morts.

Coquille ombiliquée, à moitié ouverte, déprimée, solide, marquée de stries obliques et granuleuses, granulations punctiformes, petites, parsemées par groupes. Sur la partie basale du dernier tour les granulations sont isolées et presque invisibles à l'œil nu; couleur marron; spire peu élevée; tours de spire au nombre de 5, très-peu convèxes, le dernier muni d'une carène et brièvement descendant, enflé en dessous et comprimé vers la bouche. Bouche oblique, petite, trapéziforme. Peristome épaissi, couleur pourpre-fauve, un peu canaliculé sur le bord droit, à bords joints par une couche de callus blanc, les bords supérieur et inférieur légèrement étalés et renversés, le columellaire oblique et arrondi. Une dent coniforme au-dessous de la carène.

Je dédie cette espèce à l'infatigable explorateur du Pérou, M. Stolzmann, qui a enrichi le Musée de Varsovie d'un grand nombre d'objets concernant toutes les branches d'histoire naturelle.

\section{Bulimus (Borus) popelairianus, Nyst.}

Bulimus (Borus) popelairianus, Nyst, Monog. Helic. viv. Pfr. t. ii. p. 20 ; Martens in Pfr. Novit. Conch. t. v. p. 3.

Palmal, envoyé par M. Stolzmann en une dizaine d'exemplaires, avec deux œufs de la grandeur d'un œuf de Colombe, fourni aussi en 1870 par M. Jelski en deux exemplaires de Montérico. 
8. Bulimus (Borus) sancta-Crucis, d'Orb.

Bulimus (Borus) sancta-crucis, d'Orb., Monogr. Helic. viv. Pfr. t. ii. p. 23 ; Mart. in Pfr. Novit. Conch. t. v. p. 6.

Amable Maria, envoyé par M. Jelski en 1871.

9. Bulimus (Borus) lichtensteini, Alb.

Bulimus (Borus) lichtensteinii, Alb., Monogr. Helic. viv. Pfr. t. iv. p. 366 ; Mart. in Pfr. Novit. Conch. t. v. p. 20.

Guajango, envoyé par M. Stolzmann en 1878.

10. Bulimus (Borus) moritzianus, Pfr.

Bulimus (Borus) moritzianus, Pfr., Monogr. Helic. viv. Pfr. t. ii. p. 23.

Punamarca, envoyé par M. Jelski en 1872.

11. Bulimus (Dryptus) Sangoe, Tschudi.

Bulimus (Dryptus) sangoe, Tschudi, Monogr. Helic. viv. Pfr. t. iii. p. 317.

Amable Maria, envoyé par M. Jelski en 1872, en 2 exemplaires.

12. Bulimus (Porphyrobaphe) iostomus, Sow.

Bulimus (Porphyrobaphe) iostomus, Sow., Monogr. Helic. viv. Pfr, t. ii. p. 29.

Lechugal, près de Tumbez, envoyé par M. Stolzmann en une tren. taine d'exemplaires.

13. Bulimus (Porphyrobaphe) wrzesniowskit, n. sp. (Plate LV. figs. 7, 8.)

Testa imperforata, ovato-elongata, solida, nitida, longitudinaliter striata et minutissime decussata, carneo-fulvescens, strigis longitudinalibus fuscis punctisque sordide liliaceis ornata; spira conica, obtusa; anfractibus convexiusculis, ultimus spiram aquans, basi attenuatus; columella callosa plicato-torta; apert. auriformis, basi angulata, intus alba; perist. album, crassum et expansum, marginibus callo nitidissimo albo junctis, columellari plicato adnato.

Long. 78, diam. 37 mill. Apert. cum perist. 42 mill. longa, intus 16 mill. lata, perist. 4 mill. latum.

Tambillo, envoyé par Mr. Stolzmann en 1878, en un seul exemplaire.

Coquille imperforée, solide, de forme ovoïde-allongée, luisante, spire conique à sommet obtus. Tours de spire au nombre de six, peu convexes, les deux premiers tours, de couleur carnée, sont régulièrement granulés, les deux suivants, fauves, ont des stries distantes et très-marquées, et les deux derniers sont superficiellement striés et croisés par d'autres stries presque invisibles à l'œil nu; la couleur de la coquille a, en général, une teinte carnée, le dernier tour seulement est jaune-verdâtre. Les quatre derniers tours sont marqués longitudinalement de flammes brunes et de ponctuations lilas sales. 
Columelle blanche, calleuse, munie d'un pli et tordue. Ouverture inclinée vers l'axe, anguleuse à sa base, blanche à l'intérieur, en forme d'oreille. Peristome blanc, luisant, épaissi et étalé, à bords joints par un callus se continuant à l'intérieur, bord columellaire à pli, affixe.

Je dédie ce Bulime à M. Wrzesniowski, professeur de Zoologie de l'Université de Varsovie, qui par sa science, ses travaux sur les infiniment petits, et par ses découvertes de nouveaux Crustacées, nommément de l'ordre des Amphipodes, s'est acquis une réputation dans la science. À son obligeance je dois aussi les dessins des coquilles que je joins à ces notes.

14. Bulimus (Orphnus) foveolatus, Reeve.

Bulimus (Orphnus) foveolatus, Reeve, Proc. Zool. Soc. 1849, p. 97 ; Monog. Helic. viv. Pfr. t. ii. p. 24.

Amable Maria, envoyé par M. Jelski en 1871.

15. Bulimus (Orphnus) bifasciatus, Phil.

Bulimus (Orphnus) bifasciatus, Phil., Monogr. Helic. viv. Pfr. t. ii. p. 199. $18{ }^{2} 2$.

Junin, envoyé par M. Jelski en une dizaine d'exemplaires en

16. Bulimus (Orphnus) brephoides, d'Orb.

Bulimus (Orphnus) brephoides, d'Orb., Monogr. Helic. viv. Pfr. t. ii. p. 143.

Amable Maria, envoyé par M. Jelski en 1872.

17. Bulimus (Orphnus) porphyreus, Pfr.

Bulimus (Orphnus) porphyreus, Pfr., Monogr. Helic. viv. Pfr. t. ii. p. 199.

Chota, envoyé par M. Stolzmann en une vingtaine d'exemplaires en 1877 , assez commun dans cette localité.

18. Bulimus (Orphnus) tserni, Phil.

Bulimus (Orphnus) tserni, Phil., Monogr. Helic. viv. Pfr. t. vi. p. 121.

Amable Maria, envoyé par M. Jelski en deux exemplaires en 1872.

19. Bulimus (Orphnus) Jelskir, n. sp. (Plate LVI. figs. 1, 2.)

Testa subperforata, elongato-ovata, apice obtusa, striata, translucens, saturate fulva, quadrifasciata, fasciis brunneis, superior et basalis lata, mediana et suturalis angustissima; sutura irregulariter crenulato-plicata; anfractibus 6 , convexiusculis, ultimo spiram subcquante; columella vix plicata, subtorta; apert. ovalis, oblonga, intus albescens; perist. simplex, marginibus callo tenuissime junctis, dextro recto, columellari dilatato rimam formante, livido.

Long. 35, diam. 15 mill. Apert. 17 mill. longa, 8 lata. 
Amable Maria, près de Tarma, envoyé en 1873 par M. Jelski, en un seul exemplaire.

La coquille de ce Bulime est presque close, de forme ovale-oblongue, transparente, assez solide, striée irrégulièrement, de couleur jaune-olivâtre, munie de 4 bandes brunes, dont deux plus étroites et deux plus larges. Sa spire est conique, à tours convexes et à sommet obtus ; la suture est crénelée et munie de plis courts. Tours de spire au nombre de six, le dernier égalant presque la spire. La columelle ayant un pli obsolète est légèrement tordue. Ouverture ovale-oblongue, couverte à l'intérieur d'une légère couche de callus blanc, laissant apercevoir par transparence les bandes brunes. Peristome simple, émoussé; les bords sont joints par un callus trèsmince, le bord columellaire, de couleur livide, est un peu élargi, couvrant la perforation.

Je dédie ce Bulime au savant explorateur M. Jelski, qui dans les localités qu'il a visitées, l'île de Madère, la Guyane, la Martinique, et enfin le Pérou, a laissé des traces ineffaçables de son activité et de l'attachement à sa patrie, qu'il a enrichi de ses collections, recuellies avec tant de peines et de discernement.

20. Bulimus (Goniostoma) chanchamayensis, Hidalg.

Bulimus (Goniostoma) chanchamayensis, Hidalg., Monogr. Helic. viv. Pfr. t. viii. p. 111.

Tarma, envoyé par M. Jelski en 1872, en trois exemplaires.

21. Bulimus (Drymeus) interpictus, Mart.

Bulimus (Drymaus) interpictus, Mart., Monogr. Helic. viv. Pfr. t. vi. p. 21 .

Junin, envoyé par M. Jelski en deux exemplaires en 1872 .

22. Bulimus (Drymeus) alto-peruvianus, Reeve.

Bulimus (Drymaus) alto-peruvianus, Reeve, Monogr. Helic. viv. Pfr. t. iii. p. 366 .

Tambillo, envoyé par M. Stolzmann en 1878, en une dixaine d'exemplaires.

23. Bulimus (Drymeus) cantatus, Reeve.

Bulimus (Drymaus) cantatus, Reeve, Monogr. Helic. viv. Pfr. t. iii. p. 373 .

Tarma, un seul exemplaire fourni par M. Jelski en 1872.

24. Bulimus (Drymeus) orthostoma, E. A. Smith.

Bulimus (Drymaus) orthostoma, E. A. Smith, Proc. Zool. Soc. 1877, p. 364 .

Tambillo, envoyé par M. Stolzmann en 1878, en huit exemplaires.

25. Bulimus (Scutalus) nigropileatus, Reeve.

Bulimus (Scutalus) nigropileatus, Reeve, Monogr. Helic. viv. Pfr. t. iii. p. 427 .

Chota, envoyé par M. Stolzmann en 1878, en une dizaine d'exemplaires. 
26. Bulimus (Scutalus) proteus, Brod.

Bulimus (Scutalus) proteus, Brod., Monogr. Helic. viv. Pfr. t. ii. p. 61 .

Bulimus sordidus (Desh.), Reeve.

Tarma, envoyé par M. Jelski en 1872, et par M. Stolzmann de Chota en 1878, en grand nombre et beaucoup de variétés.

27. Bulimus (Scutalus) alutaceus, Reeve.

Bulimus (Scutalus) alutaceus, Reeve, Monogr. Helic. viv. Pfr. t. iii. p. 324 .

Amable Maria, envoyé par M. Jelski en 1872, environ vingt exemplaires.

28. Bulimus (Scutalus) badius, Sow.

Bulimus (Scutalus) badius, Sow., Monogr. Helic. viv. Pfr. t. ii. p. 189.

Tarma, envoyé par M. Jelski en 1872, une dizaine d'exemplaires environ.

29. Bulimus (Scutalus) versicolor, Brod.

Bulimus (Scutalus) versicolor, Brod., Monogr. Helie. viv. Pfr. t. ii. p. 61 .

Truxillo, procuré par M. Jelski en 1871, en beaucoup d'exemplaires.

30. Bulimus (Thaumastus) chrysomelas, Mart.

Bulimus (Thaumastus) chrysomelas, Mart., Monogr. Helic.viv. Pfr. t. vi. p. 133.

Tunin, envoyé par M. Jelski en trois exemplaires en 1872 .

31. Bulimus (Obeliscus) haplostylus, Pfr.

Bulimus (Obeliscus) haplostylus, Pfr., Monogr. Helic. viv. Pfr. t. ii. p. 152 .

Tambillo, trouvés sous les feuilles par M. Stolzmann, en grand nombre, et envoyé en 1878 .

32. Bulimus (Plectostylus) косніi, Pfr.

Bulimus (Plectostylus) kochii, Pfr., Monogr. Helic. viv. Pfr. t. ii. p. 148.

Amable Maria, envoyé en 1872 par M. Jelski, en trois exemplaires.

33. Bulimus (Mesembrinus) trujillensis, Phil.

Bulimus (Mesembrinus) trujillensis, Phil., Monogr. Helic. viv. Pfr. t. vi. p. 129.

Trujillo, envoyé par M. Stolzmann en 1877, environ une dizaine d'exemplaires. 
34. Bulimus (Mesembrinus) sachsei, Alb.

Bulimus (Mesembrinus) sachsei, Alb., Monog. Helic. viv. Pfr. t. iv. p. 484.

Tambillo, envoyé par M. Stolzmann en 1878. Dix exemplaires environ.

35. Bulimus (Mesembrinus) pecilus, d’Orb. (?)

Bulimus (Mesembrinus) pacilus, d'Orb., Monog. Helic. viv. Pfr. t. ii. p. 200 .

La description de M. d'Orbigny semble s'accorder arec l'espèce envoyée par M. Stolzmann; mais elle diffère dans quelques traits particuliers aux 25 exemplaires que je possède; par le sommet de la coquille, qui n'est aucunement noir, la disposition des bandes est autre et le nombre de celles-ci est moindre, n'arrivant jamais à 7 ou 8 , et par le péristome qui est simple.

Le sommet et la coloration des bandes présentent les différences suivantes :

1. Var. sommet rose, à 6 bandes noires bordées d'orange, 1 exemplaire.

2. Var. sommet jaunâtre, à 6 bandes interrompues noires, 7 exemplaires.

3. Var. sommet corné à 3 bandes jaune-paille dans la partie supérieure de la coquille, et 3 bandes noires dans la partie basale. tion.

4. Var. sommet blanchâtre à 3 bandes noires autour de la perfora-

5. Var. sommet doré à 3 bandes noires autour de la perforation, la coquille étant jaune.

6. Var. sommet rose, coquille blanchâtre, à une bande étroite blanche au milieu du dernier tour, celui-ci étant ornée de flamme Iongitudinale jaune-paille.

Chota, envoyé par M. Stolzmann en 1878.

36. Bulimus (Leptomerus) molecillus, Reeve.

Bulimus (Leptomerus) molecillus, Reeve, Monogr. Helic. viv. Pfr. t. iii. p. 183.

Tarma, envoyé par M. Jelski en 1872.

37. Perideris flammigera, Ferr.

Perideris flammigera, Ferr., Monogr. Helic. viv. Pfr. t. ii. p. 245.

Chota, envoyée par M. Stolzmann en une trentaine d'exemplaires, en 1878 .

38. Pupa paredesil, d'Orbigny.

Pupa paredesii, d'Orbigny, Monogr. Helic. viv. Pfr. t. ii. p. 309. Chorillos, trouvée par M. Stolzmann en 1877, sous les pierres.

39. Clausilia peruana, Tschudi.

Clausilia peruana, Tschudi, Monogr. Helic. viv. Pfr. t. ii. p. 483. Mes trois exemplaires, quoique décollés, diffèrent de la descrip- 
tion faite par M. Troschel dans le 'Zeitschrift für Malacologie,' 1841 , p. 51 , en ce que deux de ceux-ci ont 9 tours de spire, et 1 seul est de 7 tours.

Tarma, envoyée par M. Jelski en 1872.

40. Clausilia taczanowskir, n. sp. (Plate LVI. figs. 3, 4.)

Testa non rimata, cylindracea, ventricosiuscula, decollata, fuliginosa, striata et costata, costis lamelliformibus distantioribus albidis undulatis et interdum irregulariter sculptis; anfr. 6-8 parum convexi, ultimus paulo angustior, fere angulatus; apert. subquadrato-ovata, fusca. Lamella supera marginalis, flexuosa; infera profundior, obliqua; subcolumellaris arcuata, subemersa; lunella valida; plica palatali unica. Perist. incrassatum, undique liberum, reflexum, fulvescens. Clausilium ovato-elongatum, apice acuminatum.

Long. 22-25‘ diam. 6 mill. Apert. 6 mill. longa, $4 \frac{1}{2}$ lata.

Bambamarca, recueillies par M. Stolzmann, le 2 mars 1878 .

Coquille non rimée, cylindrique, tant soit peu ventrue, décollée, grise, munie de stries assez élevées, particulièrement vers la suture, et de côtes lamelliformes, distantes, anguleuses, ressemblant à des lettres hébraïques, de couleur blanchâtre; tours de spire 6-8 peu convexes, le dernier tour un peu comprimé et presque anguleux à sa base. Ouverture presque carrée, un peu plus étroite vers le bas, de couleur brune. Lamelle supérieure flexueuse, atteignant le bord, l'inférieure oblique, plus profonde, lamelle columellaire arquée; lunelle forte; pli palatale allongé. Péristome réfléchi, sale.

Je dédie cette nouvelle Clausilie à Mons. Ladislas Taczanowski, ornithologiste et arachnéologue d'un grand mérite, comme homage de mon amitié et comme preuve de ma réconnaissance à l'homme, qui a bien voulu me guider de ses conseils dans mes travaux conchyliologiques.

\section{Claudilia slosarskit, n. sp. (Plate LVI. figs. 5, 6, 7.)}

Testa non rimata, cylindraceo-fusiformis, decollata, violaceo-brunnea, sericea, subtiliter et undulatim striata; anfr. 6-7, ultimus breviter solutus; cervix rotundata; apert. rotunduto-ovata, fuscescens ; lamella supera marginalis, elevata; infera obliqua; spiralis flexuosa, et subcolumellaris continua; lunella subarcuata; plica palatalis unica, elongata; perist. undique liberum, late reflexum, sordidum. Clausilium ovato-oblongum.

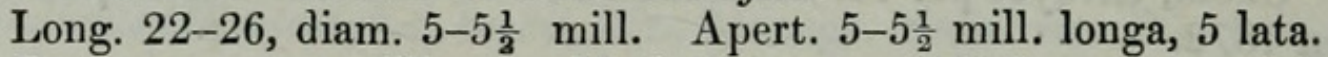

Pumamarca, recueillies par M. Stolzmann en 1878.

Coquille non rimée, fusiforme, décollée, de couleur brune-violacée, soyeuse, finement striée; les stries sont longitudinalement ondulées, les stries transversales sont distantes et obsolètes, se laissant apercevoir sur les premiers tours de quelques exemplaires. Tours de spire au nombre de $6-7$, le dernier détaché en avant et arrondi à sa base. Lamelle supérieure elevée ; l'inférieure oblique ; lamelle spirale flexueuse, et la columellaire non interrompue. Lunelle distincte, peu 
arquée. Pli palatal unique allongé. Péristome réfléchi, de couleur blanc-sale.

Je dédie cette Clausilie à l'adjoint de la chaire d'anatomie comparée, Mons. Antoine Slósarski, qui s'est occupé particulièrement de la faune conchyliologique du Royaume de Pologne, et qui par ses travaux anatomiques sur les Mollusques s'est acquis une réputation dans le pays.

42. Clausilia filocostulata, n. sp. (Plate LVI. figs. 8, 9, 10, 11.)

Testa non rimata, cylindraceo-turrita, decollata, fulvescens, tenuis, translucens, irregulariter capillaceo-lamellosa; anfr. 8, convexiusculi, ultimus solutus, breviter descendens, supra aperturam sulcatus; cervix vix rotundata; lamella supera marginalis compressa, infera vix elevata, subcolumellaris immersa; lunella brevis, arcuata ; plica palatalis unica, distincta. Perist. albidum, continuum, subexpansum. Clausilium ovato-lanceolatum.

Long. 17, diam. 4 mill. Apert. 3 mill. longa, 2 lata.

Dans la localité, nommée Escalon, entre Tunin et Obrajillo, trouvées par M. Jelski, en 1872 au mois de novembre, dans les roches calcaires.

Coquille non rimée, cylindrique et turriculée, grèle, un peu transparente, jaunâtre, munie de lamelles filiformes, les unes en groupes, les autres distantes, plus pâles que le fond; tours de spire au nombre de 8 , peu convexes, le dernier arrondi. Ouverture presque carrée, arrondie vers sa base. Lamelle supérieure marginale, comprimée; l'inférieure élevée, la columellaire grèle. Lunelle arquée, courte. Pli palatal unique et distinct. Péristome blanchâtre, continu et brièvement réfléchi.

43. Clausilia chacaënsis, n. sp. (Plate LVI. figs. 12, 13, 14,

Testa non rimata, fusiformis, solidula, cornea, oblique striatula; anfr. 9, convexi, ultimus breviter solutus, angustior, basi vix compressus, oblique elongatus; apert. trapezoideo-ovalis, lateraliter compressa; lamella supera marginalis ; infera obliqua, valida, atro-fulva; subcolumellaris immersa; lunella semicircularis, distincta; plica palatalis unica, elongata. Perist. albidum et vix incrassatum. Clausilium pyriforme.

Long. 14, diam. $2 \frac{1}{2}$ mill. Apert. 4 mill. longa, 2 lata.

Des environs de Chaca, à une heure de distance de Huanta, envoyée par M. Jelski en 1873.

Coquille non rimée, fusiforme, assez solide, striée obliquement, cornée; dans quelques exemplaires on remarque vers la suture des taches plus foncées alternant avec de plus claires; tours de spire 9, convexes, le dernier rétréci, peu prolongé et arrondi à sa base. Ouverture trapézoïdale et allongée. Lamelle supérieure marginale; l'inférieure oblique, plus forte et de couleur brune; la columellaire inmmergée ; lunelle arrondie et distincte; pli palatal allongé. Péri-

Proc. Zool. Soc.-1879, No. XLVII. 
stome grisâtrc, réflechi et épaissi. Je donne le nom à cette Clausilie de la localité où cette espèce a été trouvée.

\section{Guesteria branickit, n. sp.}

Envoyée par M. Stolzmann en 1878 de Tambillo, dont la description va incessamment paraître dans le 'Bulletin' de la Société Zoologique de France.

\section{EXPLANATION OF THE PLATES.}

\section{Plate LV.}

Figs. 1-3. Helix (Systrophia) pseudo-planorbis, p. 719.

4-6. - (Isomera) stolzmanni, p. 720.

7, 8. Bulimus (Porphyrobaphe) wrzesniowskii, p. 721.

Plate LVI.

Figs. 1, 2. Bulimus (Orphnus) jelskii, p. 722.

3, 4. Clausilia taczanowskii, p. 726 .

5-7. - slosarskii, p. 726.

8-11. filocostulata, p. 727 .

12-15. - chacaënsis, p. 727.

3. Descriptions of two new Species of Helix (Eurycratera) from S.E. Betsileo, Madagascar. By George French Angas, C.M.Z.S., F.L.S., \&c.

[Received October 27, 1879.]

\section{(Plate LVII.)}

Helix (Eurycratera) betsileoensis, n. sp. (Plate LVII. figs. 1, 2.)

Shell broadly umbilicated, depressedly ovate, rather solid, somewhat shining, irregularly transversely rugosely striated on the last whorl, the upper whorls finely and distinctly granulated, the last faintly keeled at the periphery, dark-olive brown, spotted here and there with greenish yellow, darker below the keel, with very indistinct indications of bands above it, upper whorls greenish yellow, spire flatly convex, apex depressed; whorls $3 \frac{1}{2}$, rapidly increasing; base tumid and convex around the wide umbilicus ; aperture very oblique, quadrately ovate, interior pearly grey, under the lens very minutely shagreened; peritreme thickened and slightly reflexed, columella a little expanded over the umbilicus, coarsely granulated along the entire inner surface, margins united by a callus.

Diam. maj. 3 poll. 3 lin., min. 2 poll. 6 lin., alt. 1 poll. 6 lin.

Hab. S.E. Betsileo, Madagascar.

Larger and more elongated than $H$. guesteriana, Crosse, with a tumid base and a wide umbilicus; this species wants the malleated sculpture so remarkable in $H$. guesteriana, as well as the second dark raised ridge above the periphery. It differs also from H. cornugiganteum in shape, the latter being rounder with a flattened base and a small umbilicus. 


\section{$2 \mathrm{BHL}$ Biodiversity Heritage Library}

Lubomirski, Prince Ladislas. 1879. "2. Notice sur quelques Coquilles du Pérou." Proceedings of the Zoological Society of London 1879, 719-728. https://doi.org/10.1111/j.1096-3642.1879.tb02707.x.

View This Item Online: https://www.biodiversitylibrary.org/item/90455

DOI: https://doi.org/10.1111/j.1096-3642.1879.tb02707.x

Permalink: https://www.biodiversitylibrary.org/partpdf/73446

\section{Holding Institution}

Natural History Museum Library, London

\section{Sponsored by}

Natural History Museum Library, London

\section{Copyright \& Reuse}

Copyright Status: Public domain. The BHL considers that this work is no longer under copyright protection.

This document was created from content at the Biodiversity Heritage Library, the world's largest open access digital library for biodiversity literature and archives. Visit BHL at https://www.biodiversitylibrary.org. 